\title{
Immunogenicity and efficacy after switching from original Ranibizumab to a Ranibizumab biosimilar: real-world data
}

\author{
Ashish Sharma ${ }^{1}$ M. Hafeez Faridi ${ }^{2} \cdot$ Nilesh Kumar $^{1}{ }^{1} \cdot$ Nikulaa Parachuri $^{1} \cdot$ Rohini Sharma $^{3}$. \\ Baruch D. Kuppermann ${ }^{4}$. Francesco Bandello ${ }^{5}$ Anat Loewenstein ${ }^{6} \cdot$ Carl D. Regillo $^{7}$
}

Received: 24 October 2019 / Accepted: 15 November 2019 / Published online: 16 December 2019

(c) The Royal College of Ophthalmologists 2019

Biological therapy has revolutionized the treatment of retinal vascular diseases. After the expiration of patents for biological innovator products, developments of biosimilar molecules have increased. According to the World Health Organization (WHO) definition, biosimilars are biotechnological products that are comparable with an already approved reference product in quality, nonclinical and clinical evaluation. Razumab (Intas Pharmaceuticals Ltd, Ahmedabad, GJ, India) was the first ophthalmic biosimilar approved for the similar indications as the reference product Lucentis (Ranibizumab; Genentech, South San Francisco, CA). Our aim was to understand the effect of switching from originator ranibizumab to biosimilar ranibizumab. Ranibizumab (Lucentis) has been effective for the treatment of retinal diseases such as diabetic macular oedema (DMO), retinal vein occlusion (RVO) and neovascular age-related macular degeneration (nAMD) for a decade or more. However, it comes with a high financial burden on health care systems, providers and patients. Biosimilar ranibizumab

Ashish Sharma

drashish79@hotmail.com

1 Department of Vitreoretina, Lotus Eye Hospital and Institute, Coimbatore, TN, India

2 College of Pharmacy, Chicago State University, Chicago, IL, USA

3 Department of Healthcare, The TIPS Global Institute, Coimbatore, Tamil Nadu, India

4 Gavin Herbert Eye Institute, University of California, Irvine, CA, USA

5 Department of Ophthalmology; University Vita-Salute, Scientific Institute San Raffaele, Milano, Italy

6 Division of Ophthalmology, Tel Aviv Sourasky Medical Center and Sackler Faculty of Medicine, Tel Aviv University, Tel Aviv, Israel

7 The Retina Service of Wills Eye Hospital, Mid Atlantic Retina, Philadelphia, PA, USA
(Razumab) was approved by the directorate general commercial intelligence (DGCI) India in 2015 [1]. Razumab has been widely implemented in India since then as a cost effective treatment option. Systemic biosimilar molecules such as CT-P13 have been tested for the efficacy and safety after switching from original molecule (Infliximab) [2, 3]. However, razumab being the first ranibizumab biosimilar in the world has not yet been tested in a switching scenario. In recent past, our group has shared the regulatory guidelines and immunogenicity for biosimilars from an ophthalmology perspective $[4,5]$. Safety and efficacy data for razumab has been published in past [6]. However, there were some reports of ocular inflammation with the use of razumab [7]. We feel that it is important to understand the effect of switching on immunogenicity and efficacy as we have many ophthalmic biosimilar molecules on the horizon to potentially be used in clinical practice [1]. To the best of our knowledge this is the first study that analyses the effect of switching to biosimilar in ophthalmology.

We retrospectively reviewed 30 consecutive eyes of 20 patients who were switched from original ranibizumab to biosimilar ranibizumab between Feb 2017 and Feb 2019. The methodology adhered to the tenets of the declaration of Helsinki. Institute's committee on human research approved the protocol. Informed consent was taken as per the routine protocol of the institute regarding data to be utilized for research purpose. Thirteen eyes had center involved diabetic macular oedema (Ci-DMO), 5 had branch retinal vein occlusion (BRVO), 2 had central retinal vein occlusion (CRVO) and 10 were nAMD. It was mandatory to have minimum 6 month follow-up data after switching to be included in the study. We systematically documented reasons for switch, any clinical signs of immunogenicity and efficacy after switching up to 6 months. Patient's visits were on the basis of pro re nata (PRN) protocol of observation and treatment. Clinical immunogenicity was considered positive if clinician (AS) has documented any anterior chamber inflammation (cells/flare) and/OR posterior chamber 
inflammation (vitritis/ snow banking/snow balls/retinitis/retinal vasculitis) during any of the visit after switching. Otherwise it was considered as negative. Efficacy was assessed under two categories (No change/negative efficacy) as per the discretion of the clinician (AS). Change in bestcorrected visual acuity (BCVA) and central macular thickness (CMT) on spectral-domain optical coherence tomography (SD-OCT) (Optovue Fremont, CA, USA) were the predominant factors considered for the assessment of efficacy.

All the patients were switched to biosimilar ranibizumab due to financial constraints. Mean number of original ranibizumab injection before switching was $2.43 \pm 0.50$ and mean number of biosimilar ranibizumab after switching was $2.63 \pm 0.49$. Mean ETDRS letter score was $49.5 \pm 14.1$ and $59.8 \pm 9.7$ before and at the end of 6 months after switching respectively. However, study was not powered enough to assess any difference in efficacy. Except for 3 eyes (10\%), all the eyes $(90 \%)$ were maintained on the biosimilar ranibizumab. Three eyes diagnosed with nAMD were switched to bevacizumab due to financial constraints. These 3 eyes had 2 injections of biosimilar ranibizumab each before switching to bevacizumab. None of the patient's record was documented with any of the clinical sign of immunogenicity. No change in efficacy was noted in any of the cases.

Development of biosimilars is challenging, because minor changes in the drug structure (e.g., changes in the protein by altering the amino-acid sequencing, as well as the protein folding) have the potential to modify the efficacy and safety of the drug in development. Biosimilars are new to ophthalmology and razumab was the first biosimilar of ranibizumab approved and used in ophthalmology (Approved only in India). Biosimilars are known to encounter nocebo effect (negative perception) around them [7]. Results of the present study are in agreement with the evidence from the biosimilar used in systemic diseases. The most extensive body of evidence on the safety of switching comes from studies on biosimilar infliximab, because it has been on the market longer than other biosimilar TNF inhibitors. However, there are also some first data available on the safety of switches with biosimilar etanercept (GP2015, SB4) and adalimumab (ABP501). Milassin et al. assessed 29 studies and concluded that switching from the originator to a biosimilar in patients with inflammatory bowel disease (IBD) is acceptable [2]. However, developments of new antidrug antibodies (ADA) were reported on switching. ADA can have an impact on efficacy and immunogenicity of these molecules. It is yet to be seen whether ADA assessment could be a part of studies assessing switching from originator to biosimilars in ophthalmology in future.

The study has significant limitations. It is small and retrospective and subject to bias on that basis. Furthermore, only 6 month data after switching is assessed which might not be sufficient. Assessing the real markers of immunogenicity such as antidrug antibodies (ADA) could be a better way to assess the immunogenicity. Single center and single clinician based observations adds to the limitation. However, initial observational data from this study suggests that switching to biosimilar ranibizumab does not have impact on clinical immunogenicity and efficacy in short term.

\section{Compliance with ethical standards}

Conflict of interest Ashish Sharma CONSULTANT: Novartis India, Allergan Global, Intas India, Bayer India. M Hafeez Faridi: None. Nilesh Kumar: None. Nikulaa Parachuri: None. Rohini Sharma: None. BD Kuppermann CLINICAL RESEARCH: Alcon, Alimera, Allegro, Allergan, Apellis, Clearside, Genentech, GSK, Ionis, jCyte, Novartis, Regeneron, ThromboGenics; CONSULTANT: Alimera, Allegro, Allergan, Cell Care, Dose, Eyedaptic, Galimedix, Genentech, Glaukos, Interface Biologics, jCyte, Novartis, Ophthotech, Regeneron, Revana, Theravance Biopharma. Francesco Bandello CONSULTANT: Allergan, Bayer, Boehringer- Ingelheim, Fidia Sooft, Hofmann La Roche, Novartis, NTC Pharma, Sifi, Thrombogenics, Zeiss. Anat Loewenstein CONSULTANT: Allergan, Novartis, Roche, Notal Vision, Fiorsightslabs, Beyeonics, Bayer Health Care. Carl D Regillo: None.

Publisher's note Springer Nature remains neutral with regard to jurisdictional claims in published maps and institutional affiliations.

\section{References}

1. Sharma A, Reddy P, Kuppermann BD, Bandello F, Lowenstein A. Biosimilars in ophthalmology: "Is there a big change on the horizon?". Clin Ophthalmol 2018;12:2137-43.

2. Milassin Á, Fábián A, Molnár T. Switching from infliximab to biosimilar in inflammatory bowel disease: overview of the literature and perspective. Ther Adv Gastroenterol. 2019;12:1756284819842748.

3. Jørgensen KK, Olsen IC, Goll GL, Lorentzen M, Bolstad N, Haavardsholm EA, et al. Switching from originator infliximab to biosimilar CT-P13 compared with maintained treatment with originator infliximab (NOR-SWITCH): a 52-week, randomised, double-blind, non-inferiority trial. Lancet. 2017;389:2304-16.

4. Sharma S, Khan MA, Chaturvedi A, RE-ENACT Study Investigators Group. Real-Life Clinical Effectiveness of Razumab ${ }^{\circledR}$ (the World's First Biosimilar of Ranibizumab) in Retinal Vein Occlusion: A Subgroup Analysis of the Pooled Retrospective REENACT Study. Ophthalmologica 2019;241:24-31.

5. Sharma A, Kumar N, Kuppermann BD, Bandello F, Loewenstein A. Understanding biosimilars and its regulatory aspects across the globe: an ophthalmology perspective. Br. J. Ophthalmol. (2019).

6. Sharma A, Kumar N, Kuppermann BD, et al. Biotherapeutics and immunogenicity: ophthalmic perspective. Eye (Lond). 2019; 33:1359-61. https://doi.org/10.1038/s41433-019-0434-y. Epub 2019 Apr 9.

7. Sharma A, Kumar N, Kuppermann BD, Francesco B, Lowenstein A. Ophthalmic biosimilars: lessons from India. Indian J Ophthalmol. 2019;67:1384-1385. 